\title{
ON THE RATIO OF FUZZY NUMBERS - EXACT MEMBERSHIP FUNCTION COMPUTATION AND APPLICATIONS TO DECISION MAKING
}

\author{
Bogdana STANOJEVIĆ ${ }^{a}$, Ioan DZIȚAC ${ }^{b}$, , Simona DZIȚAC $^{\mathrm{d}}$ \\ ${ }^{a}$ Mathematical Institute of the Serbian Academy of Sciences and Arts, \\ Kneza Mihaila 36, 11000 Belgrade, Serbia \\ ${ }^{b}$ Aurel Vlaicu University of Arad, Department of Mathematics and Computer Science, \\ Elena Drăgoi 2, Arad, Romania \\ ${ }^{c}$ Agora University of Oradea, Science, Piata Tineretului 8, Oradea, Romania \\ ${ }^{d}$ University of Oradea, Department of Energy Engineering, Universitatii 1, Oradea, Romania
}

Received 22 July 2015; accepted 30 August 2015

\begin{abstract}
In the present paper, we propose a new approach to solving the full fuzzy linear fractional programming problem. By this approach, we provide a tool for making good decisions in certain problems in which the goals may be modelled by linear fractional functions under linear constraints; and when only vague data are available. In order to evaluate the membership function of the fractional objective, we use the $\alpha$-cut interval of a special class of fuzzy numbers, namely the fuzzy numbers obtained as sums of products of triangular fuzzy numbers with positive support. We derive the $\alpha$-cut interval of the ratio of such fuzzy numbers, compute the exact membership function of the ratio, and introduce a way to evaluate the error that arises when the result is approximated by a triangular fuzzy number. We analyse the effect of this approximation on solving a full fuzzy linear fractional programming problem. We illustrate our approach by solving a special example - a decision-making problem in production planning.
\end{abstract}

Keywords: full fuzzy program, triangular fuzzy number, fuzzy aggregation, linear fractional programming, error approximation, decision making.

JEL Classification: C61.

\section{Introduction}

In majority economic and industrial engineering problems, one must make a decision, and the decision must be optimal. The optimization step consists in finding the best available values of an objective function over a well-defined domain. There are many different types

Corresponding author Bogdana Stanojević

E-mail: bgdnpop@mi.sanu.ac.rs 
of objective functions defined over different types of domains by using different types of constraints.

The optimization problems, where the objective function appears as a ratio of functions, form the fractional programming problems. Fractional programming is an important tool for modelling various decision processes, such as maximizing the profit/cost, volume/cost, or other quantities that measure the efficiency of a system. Fractional programming also appears in information theory, numerical analysis, decomposition algorithms for large linear systems, and in many other fields that are not necessarily economical.

In real-life problems, the consequences of decisions are known only with uncertainty, hence nobody knows in advance the exact effect of each decision. There is a variety of ways to deal with uncertainty. Fuzzy set theory, introduced by Zadeh (1965), is "a convenient and powerful way of modelling vague data without having recourse to stochastic concepts" (Slowinski 1998). Fuzzy set theory is a generalization of the conventional set theory, and it succeeds to manage the vagueness and imprecision in a strict mathematical manner. Zimmermann (1985) introduced the fuzzy concepts in mathematical programming.

Decision-making problems can be classified into two main categories with very distinct features. The first category includes the decision problems with a finite discrete set of possible alternatives, generally consisting of a small number of elements. For these decision problems, each alternative is completely known, and the decision maker generally ranks the alternatives according to a set of criteria. There is a variety of approaches that rank the alternatives with respect to some principles or desires. Many works, found in the recent literature, introduced fuzzy approaches for multiple attribute decision making. By weighting the evaluation information of each expert, Liu (2012) proposed a new way for producing the group decision-making matrix, used the maximum deviation method to calculate the attribute weights, and applied the TOPSIS method to rank the alternatives. Liu (2011) presented a multi-attribute group decision-making method based on weighted aggregation operators to solve decision problems in which both the attribute values and weights were interval-valued trapezoidal fuzzy numbers. Liu, Zhang (2011) constructed a reasonable evaluation indicator system of the agriculture informatization in China, and proposed a new evaluation method for promoting the agriculture informatization. Their model may be used to rank the orders of the different areas according to the informatization level, and to quantify the agriculture informatization of different areas according to the evaluation system based on two-tuple and relative operators. Liu, Jin (2012) proposed a decision-making method, based on weighted geometric aggregation operators, to solve the multiple attribute group decision-making problems in which the attribute values take the form of generalized interval-valued trapezoidal fuzzy numbers. Liu et al. (2012) presented a new method, based on ordered weighted harmonic averaging operators, to solve the same problem. Wei et al. (2013) investigated the multiple attribute group decision-making problems in which the attribute values take the form of triangular fuzzy information. They introduced some power aggregation operators for aggregating triangular fuzzy information, and applied them to develop some models for the given problem. Stanujkic (2013) proposed an extension of the MOORA method to use the triangular fuzzy numbers, discussed several methods for defuzzification and calculation of the distance between two fuzzy numbers, and showed how the ratio system and the reference point approach of the MOORA method can be used in fuzzy environment. Xu et al. (2014) proposed some new aggregation operators which are based on the Choquet integral and Einstein operations, and studied the relationship between them and the existing intuitionistic fuzzy Choquet aggregation operators. They also presented an approach, based on intuitionistic fuzzy Einstein Choquet integral operators, to solve the multiple attribute decision-making problems; and applied it to solve a practical decision-making problem involving the water resource management. 
The second category includes the decision problems for which each possible alternative for the decision satisfies some constraints. In order to identify the set of all possible alternatives for the decision, one needs to construct a mathematical model of the given constraints. Further, in order to find the best decision, one has to optimize an objective function over the set of the alternatives. Many examples of real-life decision-making problems that belong to this category can be found in (Murty 2015).

Bellman, Zadeh (1970) proposed the concept of decision making in a fuzzy environment, and further on many researchers adopted the concept in order to solve fuzzy linear programming problems. Pathak, Sarkar (2012) developed a supply chain fuzzy model considering aggregate production/distribution planning; and presented a fuzzy mixed-integer linear programming model for supply chain network design problems in a multi-echelon, multi-product, multi-stage with different methods of manufacturing at each stage, multi-distribution centres and multi-period supply chain planning. They applied fuzzy logic to solve the uncertain production, demand, capital and warehouse spaces with all costs being triangular fuzzy numbers. Chen, Xu (2015) studied the interval reciprocal comparison matrices, proposed a new fuzzy programming method to derive the priority vector from an interval reciprocal comparison matrix, and introduced a membership function to measure the decision maker's satisfaction degree for the priority vector derived from each interval constraint.

In our discussion, a full fuzzy model means a mathematical model whose both variables and coefficients are fuzzy quantities. Buckley, Feuring (2000) defined the full fuzzy linear optimization model (FFLP), by considering all coefficients and variables of a linear program as being fuzzy numbers. They first change the problem of maximizing the fuzzy value of the objective function into a multi-objective fuzzy linear programming problem. They introduced the fuzzy flexible programming, showed that it can be used to explore the whole undominated set for the multi-objective fuzzy linear program, and developed an evolutionary algorithm to solve the fuzzy flexible programs. Using their new algorithm, they solved two numerical problems and generated good solutions.

Later on, Hashemi et al. (2006) proposed a two-phase approach to find the optimal solution to a FFLP problem. They maximized the possibilistic mean value of the fuzzy objective function, obtained a subset of feasible solutions, and further minimized the standard deviation of the original fuzzy objective function over the constructed feasible set.

Hosseinzadeh et al. (2009) discussed the FFLP with all parameters and variables expressed by triangular fuzzy numbers. They used the concept of symmetric triangular fuzzy number, and introduced an approach to defuzzify a general fuzzy quantity. First, the fuzzy models were turned into two crisp linear problems; and with a special ranking on fuzzy numbers, the FFLPs were transformed to a multi-objective linear programming problem with crisp parameters.

Kumar et al. (2011) proposed a new method to find the fuzzy optimal solution of the same type of FFLPs. Using their method, the fuzzy optimal solution to a FFLP with no restrictions on the coefficients but equality constraints, occurring in real-life situations, can be easily obtained.

Razavi et al. (2013) extended a fuzzy version of the original data envelopment analysis models, and developed a method to solve it. The basic idea of their method was to transform the original DEA model, to an equivalent linear parametric programming model, applying the notion of alpha-cut. The linear fractional programming with decision variables expressed by fuzzy numbers was involved in the original model. Using the theoretical results introduced in the present paper, their solving approach may be extended further to solve a full fuzzy DEA model. 
Khan et al. (2013) proposed a modified version of the well-known simplex method for solving linear programming problems in a fully fuzzy environment. Their technique used a ranking function in the Gaussian elimination process. Unfortunately, they made a mistake, thus their method does not always provide a feasible optimal solution. The fallacy of their method was pointed out in (Bhardwaj, Kumar 2014).

Ezzati et al. (2015) proposed a novel algorithm to solve the FFLP problem by converting it to an equivalent multi-objective linear programming problem that is solved by the lexicographic method.

Giri et al. (2015) formulated and solved a full fuzzy fixed charge multi-item solid transportation problem, in which direct costs, fixed charges, supplies, demands, conveyance capacities and transported quantities were fuzzy in nature. They minimized the total fuzzy cost under fuzzy decision variables.

Pop, Stancu-Minasian (2008) proposed a method to solve the FFLFP problems with triangular fuzzy numbers. They used the Charnes-Cooper method to transform the linear fractional programming problem into a linear one. Then, they transformed the problem of maximizing a function with triangular fuzzy value into a deterministic multi-objective linear programming problem with quadratic constraints; and applied the extension principle of Zadeh to aggregate the fuzzy numbers, and the Kerre's method to evaluate the fuzzy constraints. Stanojevic, Stancu-Minasian (2012) introduced a new way of evaluating fuzzy inequalities, and based on it proposed a methodology for deriving an optimal solution to the FFLFP problem.

Pandian, Jayalakshmi (2013) proposed a new decomposition-restriction method, based on the decomposition principle and the denominator objective restriction method, to obtain an optimal fuzzy solution to the FFLFP problem.

Deb, De (2015) solved the FFLFP problem using graded mean integration representation method. They developed a computational algorithm to obtain an optimal solution by applying the simplex method.

As far as we know, all approaches to solving full fuzzy linear fractional programming problems, found in the literature, yield crisp solutions, or solutions expressed by triangular or trapezoidal fuzzy numbers. The goal of this paper is to introduce a novel approach that yields a fuzzy solution to the FFLFP problem, that is not necessary neither triangular nor trapezoidal fuzzy number. Thus we provide a tool for making good decisions in certain problems in which the goals may be modelled by linear fractional functions under linear constraints; and when only vague data are available. Our approach takes into consideration the discrete representation of the inverses of the exact membership functions of the products and ratios of fuzzy numbers, thus avoiding possible errors that may arise when a multiple occurrence of a variable in an arithmetic expression of fuzzy numbers is improperly handled.

The reminder of the paper is organized as follows. The basics of the fuzzy numbers arithmetic are presented in short in Section 1. Section 2 provides an exact formula for the membership function of a fuzzy number that is a ratio of sums of products of triangular fuzzy numbers with positive parameters; and presents a way to evaluate the approximation that arises when such fuzzy number is replaced by its corresponding triangular fuzzy number with the same parameters. In Section 3, we propose a novel approach to solve the FFLFP problems, based on interval's arithmetic. In order to illustrate our methodology, we solve a special example of decision making in production planning, and report the numerical results in Section 4. We also solve a problem recalled from the literature, and compare our numerical results to the existing results. Some concluding remarks and directions for future research are inserted in Section 5. 


\section{Fuzzy numbers arithmetic}

The purpose of this section is to recall some concepts which will be needed in the sequel. For more details, we refer the reader to (Uhrig, Tsoukalas 1997; Zimmermann 1996; Dubois, Prade 1987).

_ A triangular fuzzy number $\bar{Y}$ is a triplet $\left(y^{1}, y^{2}, y^{3}\right) \in R^{3}$. The membership function of $\bar{Y}$ is defined in connection with its real parameters as follows:

$$
\bar{Y}(x)=\left\{\begin{array}{ll}
\left(x-y^{3}\right) /\left(y^{2}-y^{3}\right), & x \in\left(y^{2}, y^{3}\right] \\
\left(x-y^{1}\right) /\left(y^{2}-y^{1}\right), & x \in\left(y^{1}, y^{2}\right] \\
0, & x \in\left(-\infty, y^{1}\right] \cup\left(y^{3},+\infty\right)
\end{array} .\right.
$$

The value $\bar{Y}(x)$ represents a number in $[0,1]$ that is the membership function of $\bar{Y}$ evaluated at $x$. The $\alpha$-cut of the fuzzy number $\bar{Y}$ is the interval

$$
[\bar{Y}]_{\alpha}=\left[\left(y^{2}-y^{1}\right) \alpha+y^{1},\left(y^{2}-y^{3}\right) \alpha+y^{3}\right] \text {. }
$$

The extension principle was formulated in (Zadeh 1965) in order to extend the known models implying fuzzy elements in the case of fuzzy entities. Given two fuzzy numbers $\bar{A}$ and $B$, and a real valued function $f: R \times R \rightarrow R$, the membership function of the fuzzy number $f(\bar{A}, \bar{B})$ is defined using the membership functions $\mu_{\bar{A}}$ and $\mu_{\bar{B}}$ of the fuzzy numbers $\bar{A}$ and $\bar{B}$, as follows:

$$
\mu_{f(\bar{A}, \bar{B})}(z)=\max _{f(x, y)=z}\left(\min \left(\mu_{\bar{A}}(x), \mu_{\bar{B}}(y)\right)\right) .
$$

Applying this principle the following well-known definitions of the addition and subtraction of triangular fuzzy numbers are obtained. Given two triangular fuzzy numbers $\bar{A}$ and $\bar{B}$, with real number parameters $\bar{A}=\left(a^{1}, a^{2}, a^{3}\right), \bar{B}=\left(b^{1}, b^{2}, b^{3}\right)$, we have

$$
\begin{aligned}
& \bar{A}+\bar{B}=\left(a^{1}+b^{1}, a^{2}+b^{2}, a^{3}+b^{3}\right), \\
& \bar{A}-\bar{B}=\left(a^{1}-b^{3}, a^{2}-b^{2}, a^{3}-b^{1}\right) .
\end{aligned}
$$

In what follows, we consider that the triangular fuzzy numbers $\bar{A}=\left(a^{1}, a^{2}, a^{3}\right)$ and $\bar{B}=\left(b^{1}, b^{2}, b^{3}\right)$ have non-negative endpoints. Multiplying their $\alpha$-cut intervals,

$$
\bar{A}_{\alpha}=\left[\left(a^{2}-a^{1}\right) \alpha+a^{1},\left(a^{2}-a^{3}\right) \alpha+a^{3}\right], \bar{B}_{\alpha}=\left[\left(b^{2}-b^{1}\right) \alpha+b^{1},\left(b^{2}-b^{3}\right) \alpha+b^{3}\right],
$$

we obtain the interval $[l(\alpha), r(\alpha)]$, where

$$
\begin{gathered}
l(\alpha)=\left(a^{2}-a^{1}\right)\left(b^{2}-b^{1}\right) \alpha^{2}+\left[\left(a^{2}-a^{1}\right) b^{1}+\left(b^{2}-b^{1}\right) a^{1}\right] \alpha+a^{1} b^{1} \\
r(\alpha)=\left(a^{2}-a^{3}\right)\left(b^{2}-b^{3}\right) \alpha^{2}+\left[\left(a^{2}-a^{3}\right) b^{3}+\left(b^{2}-b^{3}\right) a^{3}\right] \alpha+a^{3} b^{3} .
\end{gathered}
$$

Inverting the functions $l(\alpha)$ and $r(\alpha)$, the membership function of the fuzzy number $\bar{A} \cdot \bar{B}$ is

$$
\mu_{\bar{A} \cdot \bar{B}}(x)=\left\{\begin{array}{ll}
p^{2}-\sqrt{m^{2}+n^{2} x}, & x \in\left(a^{2} b^{2}, a^{3} b^{3}\right] \\
-q^{2}+\sqrt{t^{2}+s^{2} x}, & x \in\left(a^{1} b^{1}, a^{2} b^{2}\right] \\
0, & x \in\left(-\infty, a^{1} b^{1}\right] \cup\left(a^{3} b^{3},+\infty\right)
\end{array},\right.
$$


see for instance (Dzitac, Pop 2006). Parameters $p, m, n, q, t, s$ are real numbers, computed with respect to the parameters of the triangular fuzzy numbers $\bar{A}$ and $\bar{B}$. Moreover, $\mu_{\bar{A} \cdot \bar{B}}(x)$ increases on $\left[a^{1} b^{1}, a^{2} b^{2}\right]$ and decreases on $\left[a^{2} b^{2}, a^{3} b^{3}\right]$, and the result of the multiplication is not a triangular fuzzy number anymore.

Dividing the $\alpha$-cut intervals of the triangular fuzzy numbers $\bar{A}=\left(a^{1}, a^{2}, a^{3}\right)$ and $\bar{B}=\left(b^{1}, b^{2}, b^{3}\right)$ with positive parameters, we obtain the interval:

$$
\left[\frac{\left(a^{2}-a^{1}\right) \alpha+a^{1}}{\left(b^{2}-b^{3}\right) \alpha+b^{3}}, \frac{\left(a^{2}-a^{3}\right) \alpha+a^{3}}{\left(b^{2}-b^{1}\right) \alpha+b^{1}}\right] .
$$

Inverting the functions of $\alpha$ that describe the left and right endpoints of $[\bar{A} / \bar{B}]_{\alpha}$, the membership function of the fuzzy number $\bar{A} / B$ is given by (1).

$$
\mu_{\bar{A} / \bar{B}}(x)=\left\{\begin{array}{ll}
\left(-x+q^{2}\right) /\left(t^{2} x+s^{2}\right), & x \in\left(a^{2} / b^{2}, a^{3} / b^{1}\right] \\
\left(x-l^{2}\right) /\left(m^{2} x+n^{2}\right), & x \in\left(a^{1} / b^{3}, a^{2} / b^{2}\right] \\
0, & x \in\left(-\infty, a^{1} / b^{3}\right] \cup\left(a^{3} / b^{1},+\infty\right)
\end{array},\right.
$$

where the real parameters $p, m, n, q, t, s$ are computed starting from the parameters of the triangular fuzzy numbers $\bar{A}$ and $\bar{B}$. Moreover, the function $\mu_{\bar{A} / \bar{B}}(x)$ increases on the interval $\left[a^{1} / b^{3}, a^{2} / b^{2}\right]$, and decreases on $\left[a^{2} / b^{2}, a^{3} / b^{1}\right]$, see (Pop, Stancu-Minasian 2008).

\section{Exact membership functions to the ratio of certain fuzzy numbers}

Given the triangular fuzzy numbers $\overline{A_{1}}, \ldots, \overline{A_{n}}, \overline{B_{1}}, \ldots, \overline{B_{n}}, \overline{C_{1}}, \ldots, \overline{C_{n}}, \overline{D_{1}}, \ldots, \overline{D_{n}}$ with positive endpoints, the $\alpha$-cut interval of the fuzzy number $\sum_{i=1, n} \overline{A_{i}} \cdot B_{i}$ is the interval $[l(\alpha), r(\alpha)]$,
where

$$
\begin{aligned}
& l(\alpha)=\sum_{i=1, n}\left(\left(a_{i}^{2}-a_{i}^{1}\right)\left(b_{i}^{2}-b_{i}^{1}\right) \alpha^{2}+\left[\left(a_{i}^{2}-a_{i}^{1}\right) b_{i}^{1}+\left(b_{i}^{2}-b_{i}^{1}\right) a_{i}^{1}\right] \alpha+a_{i}^{1} b_{i}^{1}\right), \\
& r(\alpha)=\sum_{i=1, n}\left(\left(a_{i}^{2}-a_{i}^{3}\right)\left(b_{i}^{2}-b_{i}^{3}\right) \alpha^{2}+\left[\left(a_{i}^{2}-a_{i}^{3}\right) b_{i}^{3}+\left(b_{i}^{2}-b_{i}^{3}\right) a_{i}^{3}\right] \alpha+a_{i}^{3} b_{i}^{3}\right) .
\end{aligned}
$$

Let $\bar{Z}$ denote the fuzzy number $\left(\sum_{i=\overline{1, n}} \overline{A_{i}} \cdot \overline{B_{i}}\right) /\left(\sum_{i=\overline{1, n}} \overline{C_{i}} \cdot \overline{D_{i}}\right)$. Then, the $\alpha$-cut interval of $\bar{Z}$ is the interval $[l(\alpha), r(\alpha)]$, where

$$
\begin{array}{r}
l(\alpha)=\frac{\alpha^{2} \sum_{i=\overline{1, n}}\left(a_{i}^{2}-a_{i}^{1}\right)\left(b_{i}^{2}-b_{i}^{1}\right)+\alpha \sum_{i=\overline{1, n}}\left(\left(a_{i}^{2}-a_{i}^{1}\right) b_{i}^{1}+\left(b_{i}^{2}-b_{i}^{1}\right) a_{i}^{1}\right)+\sum_{i=\overline{1, n}} a_{i}^{1} b_{i}^{1}}{\alpha^{2} \sum_{i=\overline{1, n}}\left(c_{i}^{2}-c_{i}^{3}\right)\left(d_{i}^{2}-d_{i}^{3}\right)+\alpha \sum_{i=\overline{1, n}}\left(\left(c_{i}^{2}-c_{i}^{3}\right) d_{i}^{3}+\left(d_{i}^{2}-d_{i}^{3}\right) c_{i}^{3}\right)+\sum_{i=\overline{1, n}} c_{i}^{3} d_{i}^{3}}, \\
r(\alpha)=\frac{\alpha^{2} \sum_{i=\overline{1, n}}\left(a_{i}^{2}-a_{i}^{3}\right)\left(b_{i}^{2}-b_{i}^{3}\right)+\alpha \sum_{i=\overline{1, n}}\left(\left(a_{i}^{2}-a_{i}^{3}\right) b_{i}^{3}+\left(b_{i}^{2}-b_{i}^{3}\right) a_{i}^{3}\right)+\sum_{i=\overline{1, n}} a_{i}^{3} b_{i}^{3}}{\alpha^{2} \sum_{i=\overline{1, n}}\left(c_{i}^{2}-c_{i}^{1}\right)\left(d_{i}^{2}-d_{i}^{1}\right)+\alpha \sum_{i=\overline{1, n}}\left(\left(c_{i}^{2}-c_{i}^{1}\right) d_{i}^{1}+\left(d_{i}^{2}-d_{i}^{1}\right) c_{i}^{1}\right)+\sum_{i=\overline{1, n}} c_{i}^{1} d_{i}^{1}} .
\end{array}
$$


Note that the $\alpha$-cut interval of the fuzzy number $\bar{Z}$ is of the following shape

$$
\left[\frac{M \alpha^{2}+N \alpha+P}{R \alpha^{2}+S \alpha+Q}, \frac{M^{\prime} \alpha^{2}+N^{\prime} \alpha+P^{\prime}}{R^{\prime} \alpha^{2}+S^{\prime} \alpha+Q^{\prime}}\right],
$$

where the coefficients $M, N, P, R, S, Q, M^{\prime}, N^{\prime}, P^{\prime}, R^{\prime}, S^{\prime}$, and $Q^{\prime}$ are real numbers computed with respect to the parameters of the original triangular fuzzy numbers $\overline{A_{1}}, \ldots, \overline{A_{n}}, \overline{B_{1}}, \ldots, \overline{B_{n}}$, $\overline{C_{1}}, \ldots, \overline{C_{n}}, \overline{D_{1}}, \ldots, \overline{D_{n}}$.

Inverting the functions of $\alpha$ that describe the interval given in (2), we derive the exact membership function to the ratio of two fuzzy numbers that are sums of products of triangular fuzzy numbers, i.e. the concave increasing branch is one of the functions:

$$
z_{l}^{ \pm}(x)=\frac{-N+S x \pm \sqrt{(N-S x)^{2}-4(M-R x)(P-Q x)}}{2(M-R x)},
$$

and the convex decreasing branch is one of the functions:

$$
z_{r}^{ \pm}(x)=\frac{-N^{\prime}+S^{\prime} x \pm \sqrt{\left(N^{\prime}-S^{\prime} x\right)^{2}-4\left(M^{\prime}-R^{\prime} x\right)\left(P^{\prime}-Q^{\prime} x\right)}}{2\left(M^{\prime}-R^{\prime} x\right)} .
$$

The three parameters (left, top, and right) of the fuzzy number $\bar{Z}$ are:

$$
\left(\frac{\sum_{i=1, n} a_{i}^{1} b_{i}^{1}}{\sum_{i=1, n} c_{i}^{3} d_{i}^{3}}, \frac{\sum_{i=1, n} a_{i}^{2} b_{i}^{2}}{\sum_{i=1, n} c_{i}^{2} d_{i}^{2}}, \frac{\sum_{i=1, n} a_{i}^{3} b_{i}^{3}}{\sum_{i=1, n} c_{i}^{1} d_{i}^{1}}\right),
$$

and the proper expressions for $z_{l}(x)$ and $z_{r}(x)$ are chosen from $z_{l}^{ \pm}(x)$ and $z_{r}^{ \pm}(x)$, respectively, such that:

$$
z_{l}\left(\frac{\sum_{i=1, n} a_{i}^{1} b_{i}^{1}}{\sum_{i=1, n} c_{i}^{3} d_{i}^{3}}\right)=0, z_{l}\left(\frac{\sum_{i=1, n} a_{i}^{2} b_{i}^{2}}{\sum_{i=1, n} c_{i}^{2} d_{i}^{2}}\right)=1, z_{r}\left(\frac{\sum_{i=1, n} a_{i}^{2} b_{i}^{2}}{\sum_{i=1, n} c_{i}^{2} d_{i}^{2}}\right)=1, z_{r}\left(\frac{\sum_{i=1, n} a_{i}^{3} b_{i}^{3}}{\sum_{i=1, n} c_{i}^{1} d_{i}^{1}}\right)=0 .
$$

In what follows, we use the notation given in Figure 1, i.e. $z(x)$ represents the exact membership function of the fuzzy quantity $\bar{Z}$ (the convex-concave shape); and $T(x)$ represents the membership function of the triangular fuzzy number with the same endpoints $\left(y^{1}, y^{2}, y^{3}\right)$ as $\bar{Z}$ (the linear shape).

The following proposition gives a way to evaluate the approximation that arises in the case of using a triangular fuzzy number with the same parameters (5), instead of the exact fuzzy number defined by (3)-(4).

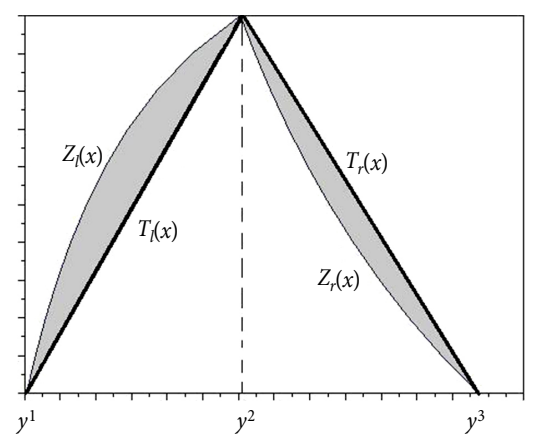

Fig. 1. The graph of the exact membership function of $Z$, and its approximation by a triangular shape 
Proposition 1. The area between the graphical representations of the membership function of the exact fuzzy number $\bar{Z}$ and the approximated triangular fuzzy number with the same parameters is equal to:

$$
\int_{0}^{1}\left|z_{l}^{-1}(\alpha)-T_{l}^{-1}(\alpha)\right| d \alpha+\int_{0}^{1}\left|z_{r}^{-1}(\alpha)-T_{r}^{-1}(\alpha)\right| d \alpha,
$$

where $z_{l}^{-1}(\alpha)$ and $z_{r}^{-1}(\alpha)$ denote the inverse of the left and right sides of $z(x)$; and $T_{l}^{-1}(\alpha)$ and $T_{r}^{-1}(\alpha)$ denote the inverse of the left and right sides of $T(x)$.

Proof. The direct way to evaluate the approximation, i.e. to compute the area of the shaded region in Figure 1, is to integrate $|Z(x)-T(x)|$ with respect to $x$, namely to compute:

$$
\int_{y_{1}}^{y_{2}}\left|z_{l}(x)-T_{l}(x)\right| d x+\int_{y_{2}}^{y_{3}}\left|z_{r}(x)-T_{r}(x)\right| d x
$$

Inverting $z(x)$ and $T(x)$ we derive $\int_{y_{1}}^{y_{2}}|z(x)-T(x)| d x=\int_{0}^{1}\left|z_{l}^{-1}(\alpha)-T_{l}^{-1}(\alpha)\right| d \alpha$ and $\int_{y_{2}}^{y_{3}}|z(x)-T(x)| d x=\int_{0}^{1}\left|z_{r}^{-1}(\alpha)-T_{r}^{-1}(\alpha)\right| d \alpha$; and integrate further the rational functions given in (2) from 0 to 1 , instead of integrating their inverses given in (3)-(4). Since both $z_{l}^{-1}, z_{r}^{-1}$ are rational functions with respect to $\alpha$, and $T_{l}^{-1}, T_{r}^{-1}$ are linear functions with respect to $\alpha$, the computation needed to evaluate the approximation is elementary.

The ratio of the fuzzy numbers introduced in this section was analysed in order to be applied to an optimization problem. Nowadays, the process of making decisions in any field cannot be competitive without an optimization step, since the optimization approaches are means for making best decisions. See (Nguyen, Kreinovich 2006), that addresses the optimization and decision making under interval and fuzzy uncertainty, and introduces some new mathematical foundations.

Mathematical programming is widely used in the natural sciences, engineering disciplines, economics, sociology and political sciences (Zimmerman 1985). Mathematical programming is one of the most frequently applied operations research techniques in solving some economic and social real-world problems. The objective functions involved in the mathematical models of real-life problems originally are non-linear. In the very beginning, only linear programming problems were solved efficiently, thus the objective functions were linearized before optimization. Even when the decision maker faced the problem of optimizing various ratios, such as profits/costs or outputs/employee, the scientists searched for linearization approaches. When the performance of the computers increased, the researchers turned back to non-linear problems and tried to solve them efficiently. Then, the field of fractional programming problems - that are the nearest generalization of the linear case - became intensively studied. As indicated in (Kornbluth, Steuer 1981), the linear fractional objectives (i.e. ratio objectives that have linear numerator and denominator) are useful in production planning, financial and corporate planning, health care and hospital planning and so forth.

Since, in most real-world applications, certainty, reliability and precision of the data is often illusory, the associated mathematical models must involve uncertain quantities, and the solving approaches must deal with the arithmetic of uncertainty.

Our next goal is to solve a full fuzzy linear fractional problem using a discrete representation of the inverses of the exact membership functions of the products and ratios of fuzzy numbers. 


\section{The FFLFP model and the solving approach}

The general model of a full fuzzy linear fractional programming (FFLFP) problem is:

$$
\begin{aligned}
& \max ^{\prime}\left(\bar{Z}=\left(\sum_{j=1}^{n} \overline{C_{j} X_{j}}+\overline{C_{0}}\right) /\left(\sum_{j=1}^{n} \overline{D_{j} X_{j}}+\overline{D_{0}}\right)\right), \\
& \text { s.t. }\left\{\begin{array}{l}
\overline{M_{i}}=\sum_{j=1}^{n} \overline{A_{i j} X_{j}} \leq \overline{B_{i}}, \quad i=1, \ldots, m \\
\overline{P_{j}} \leq \overline{X_{j}} \leq \overline{R_{j}}, \quad j=1, \ldots, n
\end{array}\right.
\end{aligned}
$$

where $\left(\overline{C_{j}}\right)_{j=1, \ldots, n}, \overline{C_{0}}$ and $\left(\overline{D_{j}}\right)_{j=1, \ldots, n}, \overline{D_{0}}$ represent the fuzzy coefficients of the objective function; $\left(\overline{A_{i j}}\right)_{i=1, \ldots, m}^{j=1, \ldots, n}$ represents the fuzzy coefficients of the left hand side of the constraints; $\left(\overline{X_{j}}\right)_{j=1, \ldots, n}$ represent the fuzzy decision variables; $\left(\overline{P_{j}}\right)_{j=1, \ldots, n}$ and $\left(\overline{R_{j}}\right)_{j=1, \ldots, n}$ represent the lower and upper bounds of the decision variables; and $\left(\overline{B_{i}}\right)_{i=1, \ldots, m}$ represent the right hand sides of the constraints. We restrict our attention to a FFLFP problem with coefficients expressed by triangular fuzzy numbers; decision variables expressed by fuzzy numbers; lower bounds on the variables expressed by fuzzy sets defined by linear increasing membership functions; and upper bounds and right hand sides of the constraints defined by linear decreasing membership functions. According to the previous section, both the objective value $\bar{Z}$ and quantities $\overline{M_{i}}, i=1, \ldots, m$ will generally not be triangular fuzzy numbers.

Existing methods for solving FFLFP problems use a simple arithmetic to aggregate triangular/trapezoidal fuzzy numbers, approximate the result by a triangular/trapezoidal fuzzy number, and then transform the original problem to a crisp multi-objective linear fractional programming problem.

Dong, Wong (1987) implemented the extension principle for computing the fuzzy weighted averages. Broek, Noppen (2011) proposed a new method to evaluate the fuzzy weighted averages of the criteria ratings in multi-criteria decision making. Based on the interval analysis (Moore 1966), both of them emphasized that the fuzzy weighted average must be treated in a different way than a general ratio of distinct fuzzy numbers, due to the multiple occurrence of the same fuzzy numbers.

We adopt their idea and use it to develop our novel approach to solve the FFLFP problems. First, we show the importance of a correct use of the fuzzy arithmetic in computing the fuzzy value of a linear fractional function with both coefficients and variables expressed by triangular fuzzy numbers.

For example, let us compute the fuzzy value of the function $\bar{Z}$,

$$
\bar{Z}(\bar{X})=\frac{(499,500,520) \cdot \bar{X}+(21,41,61)}{(2,3,11) \cdot \bar{X}+(1,2,4)},
$$

at the triangular fuzzy number $(1,2,5)$. The coefficients in the function are triangular fuzzy numbers given by their parameters (left, top, right). Ignoring the fact that the same fuzzy 
number $(1,2,5)$ appears in both nominator and denominator of $\bar{Z}$, we obtain $\bar{Z}_{\text {wrong }}$, the fuzzy number with the parameters $(8.813,130.125,887)$. A correct use of the extension principle, as pointed out by Dong, Wong (1987), yields the fuzzy number $\bar{Z}_{\text {correct }}$ with the parameters $(34.67,130.125,241.9)$.

Figure 2 shows both the correct and wrong results of applying fuzzy arithmetic. The tops of the fuzzy numbers are the same, but the support of the wrong fuzzy number is essentially wider than the support of the correct fuzzy number, thus influencing the conclusions of any analyst about the input-output relation through the function $\bar{Z}$.

Second, we present the effect of the translation of inputs, on the outputs, through a function that is a ratio of fuzzy numbers. Figure 3 shows the membership functions of the fuzzy numbers obtained by evaluating the full fuzzy linear fractional function (7) at ten points $\left(\bar{X}_{i}\right)_{i=1,10}$. The triangular shapes of the membership functions of $\left(\bar{X}_{i}\right)_{i=1,10}$ are also shown in Figure 3 - they all are equidistant translations from the triangular fuzzy number $(1,2,5)$ to the triangular fuzzy number $(4,5,8)$.

Third, we describe our novel solving approach to FFLFP problems with coefficients expressed by triangular fuzzy numbers and general fuzzy numbers as decision variables. The solving approach to FFLFP is summarized below.
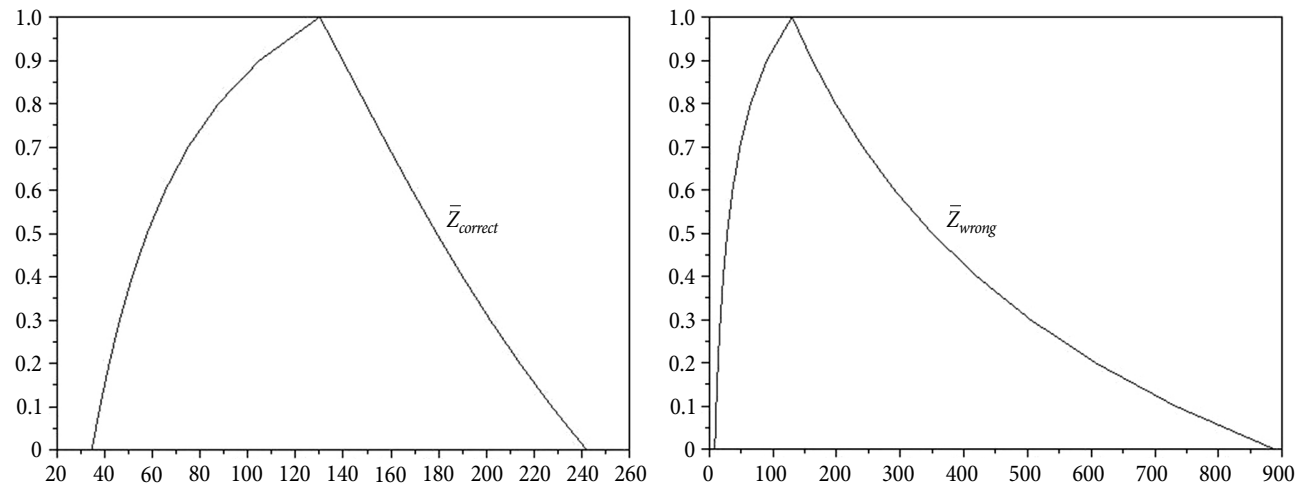

Fig. 2. Correct and wrong results in applying fuzzy arithmetic to compute the ratio $\bar{Z}$
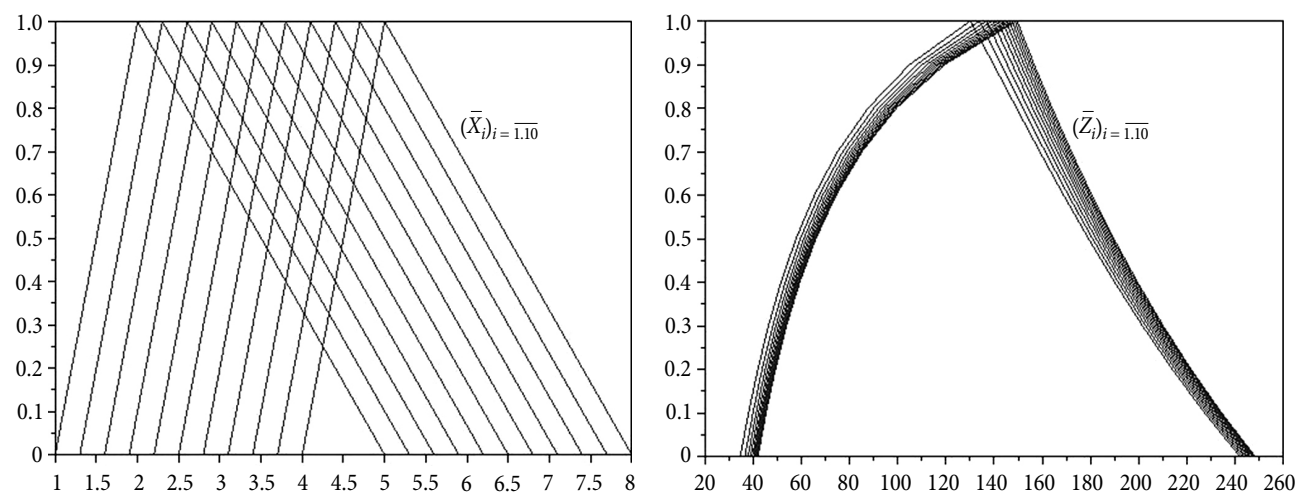

Fig. 3. The effect of the translation of the triangular fuzzy numbers $\bar{X}$ on the objective function $\bar{Z}(\bar{X})$ 
1. We decide how many $\alpha$-cuts to use in order to have a satisfactory accuracy of the membership functions of the fuzzy solution, i.e. we split the interval $[0,1]$ in $p-1$ subintervals by considering the equidistant values $0=\alpha_{1}<\ldots<\alpha_{p}=1$.

2. For each $\alpha_{i}, i=1, \ldots, p$

- we evaluate the left and right sides of the $\alpha$-cut of each fuzzy set involved in Problem (6), and solve two crisp linear fractional programming problems (8) and (9), where $Y^{L}$ and $Y^{R}$ represent the left and the right endpoints of the $\alpha_{i}$-cut interval of the fuzzy set $\bar{Y}$.

$$
\begin{aligned}
& \max \left(z=\left(\sum_{j=1}^{n} c_{j}^{L} x_{j}+c_{0}^{L}\right) /\left(\sum_{j=1}^{n} d_{j}^{R} x_{j}+d_{0}^{R}\right)\right), \\
& \text { s.t. }\left\{\begin{array}{l}
\sum_{j=1}^{n} A_{i j}^{L} x_{j} \leq B_{i}^{R}, \quad i=1, \ldots, m \\
P_{j}^{L} \leq x_{j} \leq R_{j}^{R}, \quad j=1, \ldots, n
\end{array} ;\right. \\
& \max \left(z=\left(\sum_{j=1}^{n} c_{j}^{R} x_{j}+c_{0}^{R}\right) /\left(\sum_{j=1}^{n} d_{j}^{L} x_{j}+d_{0}^{L}\right)\right), \\
& \text { s.t. }\left\{\begin{array}{l}
\sum_{j=1}^{n} A_{i j}^{R} x_{j} \leq B_{i}^{R}, \quad i=1, \ldots, m \\
P_{j}^{L} \leq x_{j} \leq R_{j}^{R}, \quad j=1, \ldots, n
\end{array}\right.
\end{aligned}
$$

- we denote by $X^{I}=\left(x_{1}^{I}, \ldots, x_{n}^{I}\right)$ and $z^{I}$ the crisp optimal solution to Problem (8), and by $X^{I I}=\left(x_{1}^{I I}, \ldots, x_{n}^{I I}\right)$ and $z^{I I}$ the crisp optimal solution to Problem (9);

- we compute $z^{L}\left(\alpha_{i}\right)=\min \left(z^{I}, z^{I I}\right), k=\arg \min \left(z^{I}, z^{I I}\right)$, and $X^{L}\left(\alpha_{i}\right)=X^{k}$ for the left sides of the $\alpha_{i}$-cut intervals of the fuzzy optimal solution; and $z^{R}\left(\alpha_{i}\right)=\max \left(z^{I}, z^{I I}\right)$, $q=\arg \max \left(z^{I}, z^{I I}\right)$, and $X^{R}\left(\alpha_{i}\right)=X^{q}$ for the right sides of the $\alpha_{i}$-cut intervals of the fuzzy solution.

Note that we treated differently the fuzzy coefficients and the fuzzy variables of Problem (6). We used only one set of variables $x=\left(x_{1}, \ldots, x_{n}\right)$ for each of the crisp problems (8) and (9), like the decision variables were not fuzzy numbers with left and right endpoints of the fuzzy intervals. The reason is that we do not know in advance what side of the fuzzy number $\bar{X}$, the left one or the right one, makes the objective function maximal. Thus, we focus on the correct construction of the $\alpha$-cut intervals of the fuzzy value $\bar{Z}(\bar{X})$, using a crisp $x$ in the optimization step; and, only in the end, decide whether the obtained optimal $x$ should be placed on the left or on the right side of the $\alpha$-cut interval of the fuzzy solution $\bar{X}$.

In our approach, we do not need any special definition for the inequality of two fuzzy numbers. We only impose on the decision variables to lie in the interval defined by their lower and upper bounds, derived from the $\alpha$-cuts of the fuzzy numbers $\bar{P}_{j}$ and $\bar{R}_{j}, j=1, \ldots, n$.

Comparing to other solving methods, our procedure has some advantages. It takes into consideration the multiple occurrences of the variables in the arithmetic expressions of the objective function and constraints, and handles it properly. It uses the discrete representation of the inverses of the exact membership functions of the products and ratios of fuzzy 
numbers. It yields fuzzy solutions expressed by general fuzzy numbers, that are not necessary neither triangular nor trapezoidal fuzzy numbers. The supports of the fuzzy solutions are the same as in the case of the crisp decision variables used instead of the fuzzy decision variables. It is easy to be implemented since it does not relay on any special definition of fuzzy inequality.

\section{Computational results}

\subsection{Numerical example}

In this section we illustrate our novel approach by solving a special example - a decisionmaking problem in production planning.

A company produces two kinds of products. The selling price for each product can vary a little due to smaller discounts to certain customers, but the unit prices are about 500 euros for the first product and 40 euros for the second one. The time needed to process each of them is approximately 3 hours for the first product and 10 hours for the second one. The fixed time needed to organize the whole production is about 20 hours. The volumes of the main substance needed are about 2 litres per unit for both products. The available main substance for the production can vary slightly from week to week. The minimum volume available per week is 55 litres, and the maximum is 75 . The minimal number of the products of the first kind is about 5 , and the maximal number is about 105 . The minimal number of the products of the second kind is about 3, and the maximal number is about 50. The company wants to determine the number of units to produce for each product per week to maximize its revenue and minimize the total production time. Since all the numbers given are uncertain, we model the problem as a FFLFP. We substitute a triangular fuzzy number for each given value, such that the top of the fuzzy number is at the given number. Hence, we have to solve the following FFLFP problem

$$
\begin{aligned}
& \max \left(\bar{Z}=\frac{(490,500,520) \cdot \bar{X}_{1}+(30,40,70) \cdot \bar{X}_{2}}{(2,3,11) \cdot \bar{X}_{1}+(8,10,21) \cdot \bar{X}_{2}+(10,20,40)}\right), \\
& \text { s.t. }\left\{\begin{array}{l}
(1,2,3) \cdot \bar{X}_{1}+(1,2,4) \cdot \bar{X}_{2} \leq(55,55,75), \\
(2,5,5) \leq \bar{X}_{1} \leq(105,105,121),
\end{array}\right. \\
& (2,3,3) \leq \bar{X}_{2} \leq(50,50,56) .
\end{aligned}
$$

Applying the methodology introduced in Section 2, we obtained the following results.

The membership functions of the maximal $X_{1}$ and $X_{2}$ for Problem (10) are shown in Figure 4. The membership function of $\bar{Z}_{\max }$ is shown on the left side of Figure 6 . Only for the exemplification purpose, we also report the results obtained by minimizing the same objective. The membership functions of the minimal $\bar{X}_{1}$ and $\bar{X}_{2}$ are shown in Figure 5, while the membership function of $\bar{Z}_{\min }$ is shown on the right side of Figure 6 .

We solved Problem (10) with two accuracy levels. First time we used $11 \alpha$-cuts, and the obtained values are represented in Figures 4, 5 and 6 by black rhombuses " $"$ ". Second time we computed the values of the membership functions for $101 \alpha$-cuts, and represented them by signs " + " in Figures 4, 5 and 6 . The effect of the number of $\alpha$-cuts used in computation, on the accuracy of the solution, can be seen in the graphical representation. 

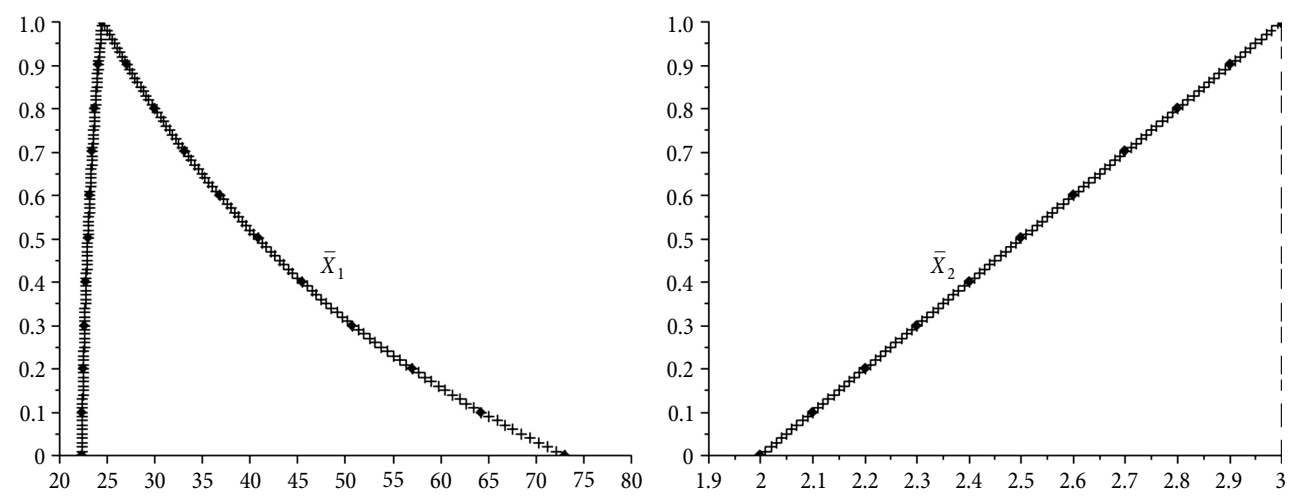

Fig. 4. The shapes of the maximal solutions $\bar{X}_{1}$ and $\bar{X}_{2}$ to Problem (10)
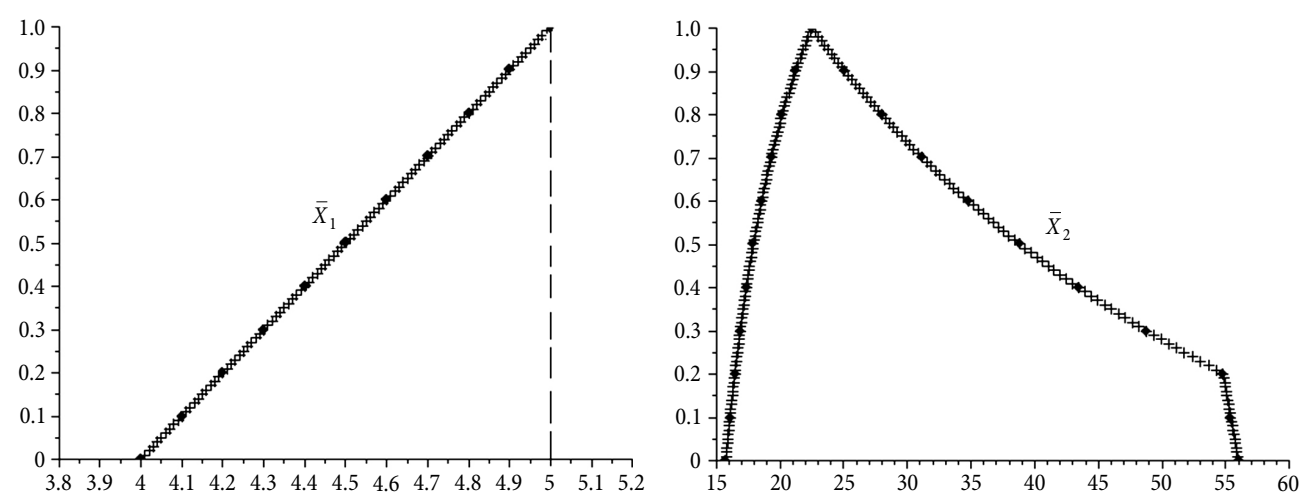

Fig. 5. The shapes of the minimal $\bar{X}_{1}$ and $\bar{X}_{2}$
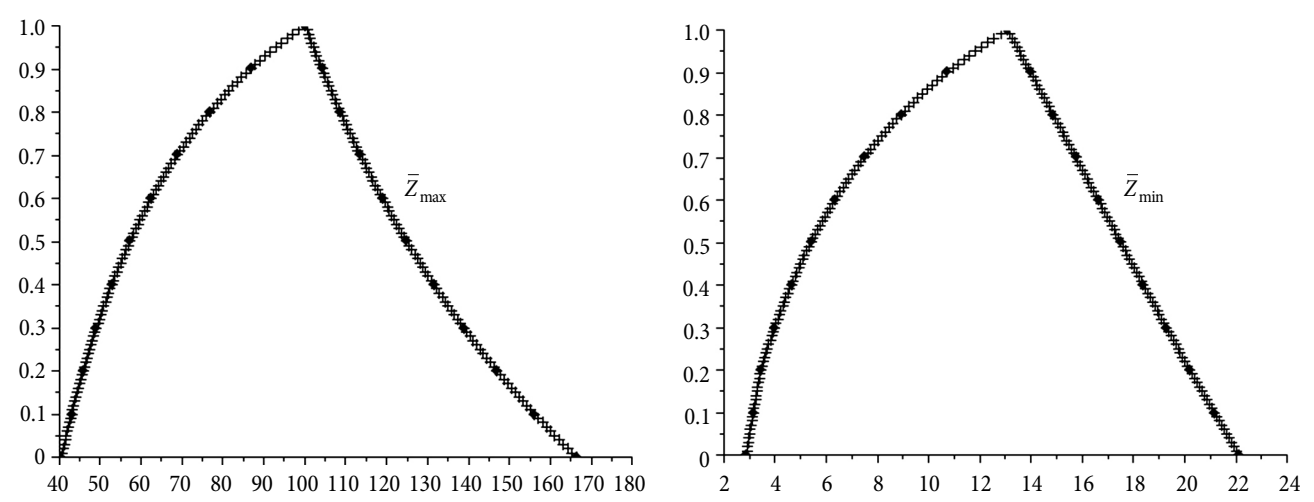

Fig. 6 . The shapes of $\bar{Z}_{\max }$ (for the maximal $\bar{X}_{1}, \bar{X}_{2}$ ) and $\bar{Z}_{\min }$ (for the minimal $\bar{X}_{1}, \bar{X}_{2}$ ) 


\subsection{Comparison to other methods}

In this section, we recall the problem solved in (Stanojevic, Stancu-Minasian 2012, 2009; Pop, Stancu-Minasian 2008):

$$
\begin{aligned}
& \max \left(\bar{Z}=\frac{(0,1,2) \cdot \bar{X}_{1}+(-2,-1,0) \cdot \bar{X}_{2}+(0,1,2)}{(0,1,2) \cdot \bar{X}_{1}+(0,1,2) \cdot \bar{X}_{2}+(1,2,3)}\right), \\
& \text { s.t. }\left\{\begin{array}{l}
(0,1,2) \cdot \bar{X}_{1}+(0,1,2) \cdot \bar{X}_{2} \leq(1,2,3), \\
(0,1,2) \cdot \bar{X}_{1}+(-2,-1,0) \cdot \bar{X}_{2} \leq(0,1,2), \\
\bar{X}_{1}, \bar{X}_{2} \geq(0,0,0) .
\end{array}\right.
\end{aligned}
$$

In (Pop, Stancu-Minasian 2008), the authors also reported the results obtained using their general method but working with crisp decision variables. In (Stanojevic, Stancu-Minasian 2012), the authors reported the solutions obtained to a similar problem that had non-symmetric triangular fuzzy numbers as coefficients, but the same top and left parameters as the triangular fuzzy coefficients of Problem (11). The numerical results are summarized in Table 1.

Figure 7 shows the optimal solution $\bar{X}_{1}$, and the optimal fuzzy value $\bar{Z}_{\max }$ obtained by applying our method to solve both Problem (11) and its variant introduced in (Stanojevic; Stancu-Minasian 2012). Dashed lines were used to show the results obtained in the case of non-symmetric triangular fuzzy coefficients. The parameters of the optimal $\bar{X}_{2}$ were $(0,0$, 0 ) in both cases, hence its graphical representation does not bring any significance to our discussion. For the optimal $\bar{X}_{1}$ and $\bar{Z}_{\max }$, it is interesting to see the change of the shapes with respect to the supports of the fuzzy coefficients of the problem.

Analysing the numerical results shown in Table 1, the following advantages of our method deserve to be mentioned:

- the increase of the values of the right parameters of the fuzzy coefficients of Problem (11) increases the values of the right parameters of the solution, and dilates the shapes of both increasing and decreasing branches of the fuzzy solutions (note that this is not the case of the method introduced in (Stanojevic, Stancu-Minasian 2012), where even the top of the fuzzy value of the objective function changed);
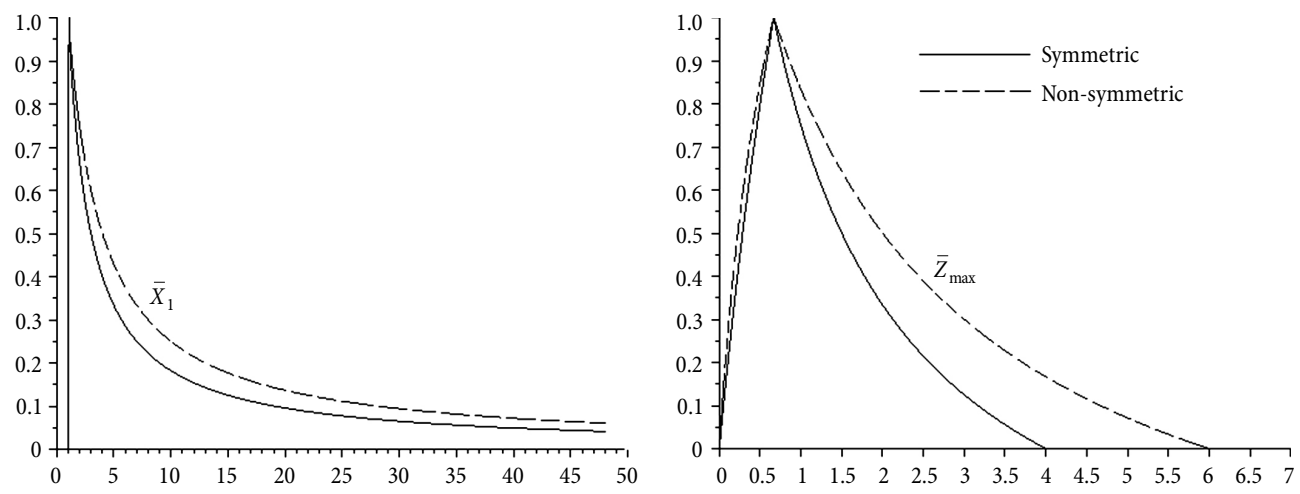

Fig. 7. The shapes of the optimal solution $\bar{X}_{1}$ and $\bar{Z}_{\max }$ to Problem (11) 
Table 1. The solutions to Problem (10) for comparison

\begin{tabular}{|c|c|c|c|c|}
\hline $\begin{array}{l}\text { Solving method/ solution } \\
\text { features }\end{array}$ & $\begin{array}{l}\text { Variables } \\
\text { features }\end{array}$ & Optimal $X_{1}$ & Optimal $\bar{X}_{2}$ & $\bar{Z}_{\max }$ \\
\hline \multirow{2}{*}{$\begin{array}{l}\text { (Pop, Stancu-Minasian } \\
\text { 2008)/triangular } \\
\text { fuzzy numbers }\end{array}$} & $\begin{array}{l}\text { triangular fuzzy } \\
\text { numbers }\end{array}$ & $(0.0047,1,1.91)$ & $(0,0,0.911)$ & $(-0.21,0.67,5.82)$ \\
\hline & crisp numbers & 1 & 0 & $(0,0.667,4)$ \\
\hline $\begin{array}{l}\text { (Stanojevic, } \\
\text { Stancu-Minasian } \\
\text { 2009)/ triangular } \\
\text { fuzzy numbers }\end{array}$ & $\begin{array}{l}\text { triangular fuzzy } \\
\text { numbers }\end{array}$ & $(0.125,1, \infty)$ & $(0,0,0)$ & $(0,0.667, \infty)$ \\
\hline \multirow{2}{*}{$\begin{array}{l}\text { (Stanojevic, } \\
\text { Stancu-Minasian } \\
\text { 2012)/ triangular } \\
\text { fuzzy numbers }\end{array}$} & $\begin{array}{l}\text { symmetric fuzzy } \\
\text { coeff. }\end{array}$ & $(0,1,1)$ & $(0,0,0)$ & $(0,0.667,1.33)$ \\
\hline & $\begin{array}{l}\text { non-symmetric } \\
\text { fuzzy coeff. }\end{array}$ & $(0,1,1)$ & $(0,0,0)$ & $(0,0.55,1.09)$ \\
\hline \multirow{2}{*}{$\begin{array}{l}\text { The new method/ } \\
\text { non-triangular } \\
\text { fuzzy numbers }\end{array}$} & $\begin{array}{l}\text { symmetric fuzzy } \\
\text { coeff. }\end{array}$ & $(1,1, \infty)$ & $(0,0,0)$ & $(0,0.667,4)$ \\
\hline & $\begin{array}{l}\text { non-symmetric } \\
\text { fuzzy coeff. }\end{array}$ & $(1,1, \infty)$ & $(0,0,0)$ & $(0,0.667,6)$ \\
\hline
\end{tabular}

- even for an optimal $\bar{X}_{1}$ with an unbounded value of the right parameter, the corresponding value $\bar{Z}_{\max }$ has a bounded support (note that this is not the case of the method introduced in (Stanojevic, Stancu-Minasian 2009));

- the support of the fuzzy value $\bar{Z}_{\max }$, obtained by our method, is the same with the support of the fuzzy value obtained by solving Problem (11) with crisp decision variables instead of fuzzy decision variables (note again that this is not the case of the other methods).

We did not include in our discussion the method introduced in (Deb, De 2015), since the numerical results reported there contain obvious mistakes: the fuzzy numbers representing the solution had the values of the parameters improper ordered, or their solutions were not proper fuzzy numbers.

\section{Conclusions}

In this paper, we developed a novel approach to solving the full fuzzy linear fractional programming problem, thus providing a tool for making good decisions in certain problems in which the goals may be modelled by linear fractional functions under linear constraints; and when only vague data are available. To the best of our knowledge, all approaches to solving full fuzzy linear fractional programming problems found in the literature yield crisp solutions, or solutions expressed by triangular or trapezoidal fuzzy numbers. On the contrary, following our methodology, the approximation of the results by triangular/ trapezoidal fuzzy numbers, and the comparison of fuzzy numbers were avoided. Moreover, comparing to existing methods, our approach used the discrete representation of the inverses of the exact membership functions of the products and ratios of fuzzy numbers, 
thus avoiding possible errors that may arise when a multiple occurrence of a variable in an arithmetic expression of fuzzy numbers is improper handled. The approach does not use any special definition of fuzzy inequality, and it is easy to be implemented.

In order to construct the membership function of the fractional objective, we used the $\alpha$-cut intervals of the fuzzy numbers obtained as sums of products of triangular fuzzy numbers with positive support. We derived the $\alpha$-cut intervals of the ratio of such fuzzy numbers, computed the exact membership function of the ratio, and presented a way to evaluate the error that arose when the result was approximated by a triangular fuzzy number. We also analysed the effect of this approximation on solving full fuzzy linear fractional programming problem, and discussed some potential applications to decision making.

The membership functions of the solution to full fuzzy linear fractional programming problem derived in this paper is discrete and its accuracy can be tuned by choosing a convenient number of $\alpha$-cut intervals to be computed. Involving the value $\alpha$ as a parameter in Problems (8) and (9), and developing a parametric analysis of the solutions to Problems (8) and (9) is a way to derive analytical expressions for the membership functions of the fuzzy solutions to full fuzzy linear fractional programming problems. Exploring this idea will be the subject of our further work.

\section{Acknowledgements}

This research was partially supported by the Ministry of Education and Science, Republic of Serbia, project number TR36006. The authors want to express their gratitude to the anonymous referees for their valuable suggestions.

\section{References}

Bellman, R. E.; Zadeh, L. A. 1970. Decision making in a fuzzy environment, Management Science 17: 141-164. http://dx.doi.org/10.1287/mnsc.17.4.B141

Bhardwaj, B.; Kumar, A. 2014. A note on the paper "A simplified novel technique for solving fully fuzzy linear programming problems", Journal of Optimization Theory and Applications 163(2): 685-696. http://dx.doi.org/10.1007/s10957-013-0505-3

Broek, P.; Noppen, J. 2011. Exact membership functions for the fuzzy weighted average, Chapter 6 in K. Madani, A. D. Correia, A. Rosa, J. Filipe (Eds.). Computational intelligence. Berlin, Heidelberg: Springer. http://dx.doi.org/10.1007/978-3-642-20206-3_6

Buckley, J. J.; Feuring, T. 2000. Evolutionary algorithm solution to fuzzy problems: fuzzy linear programming, Fuzzy Sets and Systems 109(1): 35-53. http://dx.doi.org/10.1016/S0165-0114(98)00022-0

Chen, L.; Xu, Z. 2015. A new fuzzy programming method to derive the priority vector from an interval reciprocal comparison matrix, Information Sciences 316: 148-162.

http://dx.doi.org/10.1016/j.ins.2015.04.015

Deb, M.; De, P. K. 2015. Optimal solution of a fully fuzzy linear fractional programming problem by using graded mean integration representation method, Applications and Applied Mathematics 10(1): 571-587.

Dong, W. M.; Wong, F. S. 1987. Fuzzy weighted averages and implementation of the extension principle, Fuzzy Sets and Systems 21: 183-199. http://dx.doi.org/10.1016/0165-0114(87)90163-1

Dubois, D.; Prade, H. 1987. Fuzzy numbers: an overview, Chapter 1, Vol. 1, in J. C. Bezdek (Ed.). Analysis of fuzzy information. Boca Raton: CRC Press. 
Dzitac, I.; Pop, B. 2006. On triangular fuzzy numbers arithmetic approximations in linear optimization, Bulletin of the Transilvania University of Brasov, Ser. B 48: 15-20.

Ezzati, R.; Khorram, E.; Enayati, R. 2015. A new algorithm to solve fully fuzzy linear programming problems using the MOLP problem, Applied Mathematical Modelling 39(12): 3183-3193. http://dx.doi.org/10.1016/j.apm.2013.03.014

Giri, P. K.; Maiti, M. K.; Maiti, M. 2015. Fully fuzzy fixed charge multi-item solid transportation problem, Applied Soft Computing 27: 77-91. http://dx.doi.org/10.1016/j.asoc.2014.10.003

Hashemi, S. M.; Modarres, M.; Nasrabadi, E.; Nasrabadi, M. M. 2006. Fully fuzzified linear programming, solution and duality, Journal of Intelligent and Fuzzy Systems 17(3): 253-261.

Hosseinzadeh L. F.; Allahviranloo, T.; Alimardani J. M.; Alizadeh, L. 2009. Solving a full fuzzy linear programming using lexicography method and fuzzy approximate solution, Applied Mathematical Modelling 33(7): 3151-3156. http://dx.doi.org/10.1016/j.apm.2008.10.020

Khan, I. U.; Ahmad, T.; Maan, N. 2013. A simplified novel technique for solving fully fuzzy linear programming problems, Journal of Optimization Theory and Applications 159(2): 536-546. http://dx.doi.org/10.1007/s10957-012-0215-2

Kornbluth J. S. H.; Steuer, R. E. 1981. Multiple objective linear fractional programming, Management Science 27(9): 1024-1039. http://dx.doi.org/10.1287/mnsc.27.9.1024

Kumar, A.; Kaur, J.; Singh, P. 2011. A new method for solving fully fuzzy linear programming problems, Applied Mathematical Modelling 35(2): 817-823. http://dx.doi.org/10.1016/j.apm.2010.07.037

Liu, P. D. 2011. A weighted aggregation operators multi-attribute group decision-making method based on interval-valued trapezoidal fuzzy numbers, Expert Systems with Applications 38(1): 1053-1060. http://dx.doi.org/10.1016/j.eswa.2010.07.144

Liu, P. D. 2012. An approach to group decision making based on 2-dimension uncertain linguistic information, Technological and Economic Development of Economy 18(3): 424-437. http://dx.doi.org/10.3846/20294913.2012.702139

Liu, P. D.; Jin, F. 2012. A multi-attribute group decision-making method based on weighted geometric aggregation operators of interval-valued trapezoidal fuzzy numbers, Applied Mathematical Modelling 36(6): 2498-2509. http://dx.doi.org/10.1016/j.apm.2011.09.006

Liu, P. D.; Zhang, X. 2011. Investigation into evaluation of agriculture informatization level based on two-tuple, Technological and Economic Development of Economy 17(1): 74-86. http://dx.doi.org/10.3846/13928619.2011.554007

Liu, P. D.; Zhang, X.; Jin, F. 2012. A multi-attribute group decision-making method based on intervalvalued trapezoidal fuzzy numbers hybrid harmonic averaging operators, Journal of Intelligent and Fuzzy Systems 23(5): 159-168.

Moore, R. E. 1966. Interval analysis. Englewood Cliffs, New Jersey: Prentice-Hall.

Murty, K. G. (Ed.) 2015. Case studies in operations research - applications of optimal decision making, International Series in Operations Research \& Management Science, Vol. 212. New York: Springer.

Nguyen, H. T.; Kreinovich, V. 2006. Optimization and decision making under interval and fuzzy uncertainty: towards new mathematical foundations, Studies in Fuzziness and Soft Computing 201: 275-290. http://dx.doi.org/10.1007/3-540-33517-X_10

Pandian, P.; Jayalakshmi, M. 2013. On solving linear fractional programming problems, Modern Applied Science 7(6): 90-100.

Pathak, S.; Sarkar, S. 2012. A fuzzy optimization model to the aggregate production/distribution planning decision in a multi-item supply chain network, International Journal of Management Science and Engineering Management 7(3): 163-173.

Pop, B.; Stancu-Minasian, I. M. 2008. A method of solving fully fuzzified linear fractional programming problems, Journal of Applied Mathematics and Computing 27: 227-242.

http://dx.doi.org/10.1007/s12190-008-0052-5 
Razavi, S. H.; Amoozad, H.; Zavadskas, E. K.; Hashemi, S. S. 2013. A fuzzy data envelopment analysis approach based on parametric programming, International Journal of Computers Communication and Control 8(4): 594-607. http://dx.doi.org/10.15837/ijccc.2013.4.580

Slowinski, R. 1998. Fuzzy sets in decision analysis, operations research and statistics. Kluwer Academic Publishers. http://dx.doi.org/10.1007/978-1-4615-5645-9

Stanojevic, B.; Stancu-Minasian, I. M. 2009. On solving fully fuzzified linear fractional programs, Advanced Modeling and Optimization 11(4): 503-523.

Stanojevic, B.; Stancu-Minasian, I. M. 2012. Evaluating fuzzy inequalities and solving fully fuzzified linear fractional program, Yugoslav Journal of Operations Research 22: 41-50. http://dx.doi.org/10.2298/YJOR110522001S

Stanujkic, D. 2013. An extension of the MOORA method for solving fuzzy decision making problems, Technological and Economic Development of Economy 19(Supplement 1): S228-S255. http://dx.doi.org/10.3846/20294913.2013.880083

Uhrig, R. E.; Tsoukalas, L. H. 1997. Fuzzy and neural approaches in engineering. New York: John Wiley and Sons Inc.

Wei, G.; Zhao, X.; Wang, H.; Lin, R. 2013. Fuzzy power aggregation operators and their application to multiple attribute group decision making, Technological and Economic Development of Economy 19(3): 377-396. http://dx.doi.org/10.3846/20294913.2013.821684

Xu, Y.; Wang, H.; Merigo, J. M. 2014. Intuitionistic fuzzy Einstein Choquet integral operators for multiple attribute decision making, Technological and Economic Development of Economy 20(2): 227-253. http://dx.doi.org/10.3846/20294913.2014.913273

Zadeh, A. 1965. Fuzzy sets, Information and Control 8: 338-353. http://dx.doi.org/10.1016/S0019-9958(65)90241-X

Zimmermann, H.-J. 1985. Applications of fuzzy sets theory to mathematical programming, Information Sciences 36: 29-58. http://dx.doi.org/10.1016/0020-0255(85)90025-8

Zimmermann, H.-J. 1996. Fuzzy set theory. Boston: Kluwer Academic Publishers.

Bogdana STANOJEVIĆ, obtained her doctoral degree in Mathematics in 2003 from the Romanian Academy. Currently she is researcher at Mathematical Institute of the Serbian Academy of Sciences and Arts. She authored and co-authored more than 40 scientific papers in the field of Operations Research. Her research interests include different aspects of fuzzy optimization, multiple objective optimization, and fractional programming.

Ioan DZIȚAC, obtained his doctoral degree in Information Sciences in 2002 from the Babes-Bolyai University of Cluj-Napoca, Romania. Currently he is professor at Aurel Vlaicu University of Arad, and rector of Agora University of Oradea. He authored and co-authored more than 60 scientific papers in journals and conference proceedings. His research interests include different aspects of Distributed Systems, Fuzzy Logic Applications and Artificial Intelligence.

Simona DZIT,AC, obtained her doctoral degree in Energetic Engineering in 2007 from the University of Oradea. Currently she is an associate professor at University of Oradea. She authored and coauthored more than 40 scientific papers in journals and conference proceedings. Her research interests include different aspects of Fuzzy Methods and Monte Carlo Methods in Reliability. 